\title{
An inexpensive air stream temperature controller and its use to facilitate temperature-controlled behavior in Drosophila
}

Ryan Sangston' \& Jay Hirsh",

\section{ABSTRACT}

Controlling the environment of an organism has many biologically relevant applications. Temperature-dependent inducible biological reagents have proven invaluable for elucidating signaling cascades and dissection of neural circuits. Here we develop a simple and affordable system for rapidly changing temperature in a chamber housing adult Drosophila melanogaster. Utilizing flies expressing the temperatureinducible channel dTrpA1 in dopaminergic neurons we show rapid and reproducible changes in locomotor behavior. This device should have wide application to temperature-modulated biological reagents.

\section{METHOD SUMMARY}

We develop a widely applicable and affordable solution to rapidly changing temperature within an enclosed chamber using commercially available components.

\section{KEYWORDS:}

dopamine - Drosophila • inducible $\cdot$ locomotor behavior - thermogenetics • TrpA1

'University of Virginia, Department of Biology, Charlottesville, VA, USA; ${ }^{\star}$ Author for correspondence: jh6u@virginia.edu

BioTechniques 66: 159-161 (March 2019) 10.2144/btn-2018-0152
Conditional manipulation of effector genes has improved our ability to dissect signaling pathways and neural circuits in living animals. Widely used methods of conditional manipulation include chemical-, light- and temperature-dependent tools [1]. In Drosophila melanogaster, temperature dependent tools such as the shibire ${ }^{\text {ts }}$ allele [2], Gal80 8 ts [3,4], and UAS-dTrpA1 [5] are commonly used. A key benefit afforded by conditional tools is that they allow for temporal control in a constant genetic background. A conditional gene product that is optimal for behavioral studies should be rapidly acting and reversible. Having temporal control affords additional benefits, such as studying genes that may be critical to the viability of an organism during development but disposable later in life.

Early condition expression utilized the native heat shock promoter hsp70 [6]. With the advent of more sophisticated temperature-inducible tools, such as heat-activated cation channel $d \operatorname{TrpA} 1$, dissecting neural circuitry can be accomplished. In Drosophila, the GAL4/UAS binary expression system [4] allows ectopic expression of these inducible channels in subsets of neurons to measure the physiological and behavioral alteration after temperature change. One of the first behavioral alterations showed paralysis after inducing $\mathrm{dTrpA} 1$ pan-neuronally with the C155-GAL4 driver [5]. Since then, many studies have used $\mathrm{dTrpA} 1$ to define neuronal circuits, such as those involved in learning and memory $[7,8]$, neuropeptide $F$ signaling [9], and activity levels during free run [10]. Recently, a massive screen used 2204 GAL4 lines to identify neurons responsible for sensory processing, locomotor control, courtship, aggression and sleep [11]. Despite its widespread usage, methods for generating the temperature shifts vary wildly between laboratories, and often involve physically moving the animals between environments set at the different temperatures, which itself can cause behavioral effects due to mechanical stimulation.
In our efforts to measure activity levels of free running flies while conditionally activating dopamine neurons with $\mathrm{dTrpA}$, we found three issues: first, devices that change temperature in situ are often prohibitively expensive. We therefore resorted to physically moving animals between chambers set at different temperatures. This led to our second problem, finding confounding phenotypes from mechanical agitation. Third, published methods for changing temperature are often slow $[5,12]$.

To address these issues, we developed an inexpensive device that allows rapid temperature changes in a $0.13 \mathrm{~m}^{3}$ fly chamber, which is itself housed in a constant environment room (Figure 1). As shown here, temperature shifts are rapid and reproducible, resulting in highly reliable behavioral measurements.

To demonstrate the effectiveness of our system, we measured locomotor responses subsequent to activation of $\mathrm{dTrpA} 1$ in most dopamine neurons, using flies containing the TH-GAL4 driver [13] and UAS-dTrpA1 (Figure 2). Flies were housed for at least 1 day at $21^{\circ} \mathrm{C}, 60 \%$ relative humidity $(\mathrm{RH})$, prior to temperature shifts with a 12-h light/ dark schedule, then exposed to 20 -min alternating intervals of 29 and $22^{\circ} \mathrm{C}$ in constant darkness but during subjective daytime. A probe within the chamber indicates that sequential temperature shifts are rapid and highly reproducible (Figure 2), with significant temperature increases detectable by the second minute after heater activation, then stabilizing at an average temperature of $28.7 \pm 0.6^{\circ} \mathrm{C}$ SEM for the remaining $18 \mathrm{~min}$. Temperature decreases are slightly slower due to cooling controlled solely by expulsion of heated air through the chamber vent, with a half time of $\sim 3 \mathrm{~min}$. By the fourth minute of the cooling cycle, the temperature falls to under $24^{\circ} \mathrm{C}$, at which point $\mathrm{dTrpA} 1$ is no longer induced. Activity data from the UAS-dTrpA1, TH-GAL4 flies in response to the temperature shifts is rapid and highly reproducible (Figure 2). Consistent with dopamine 


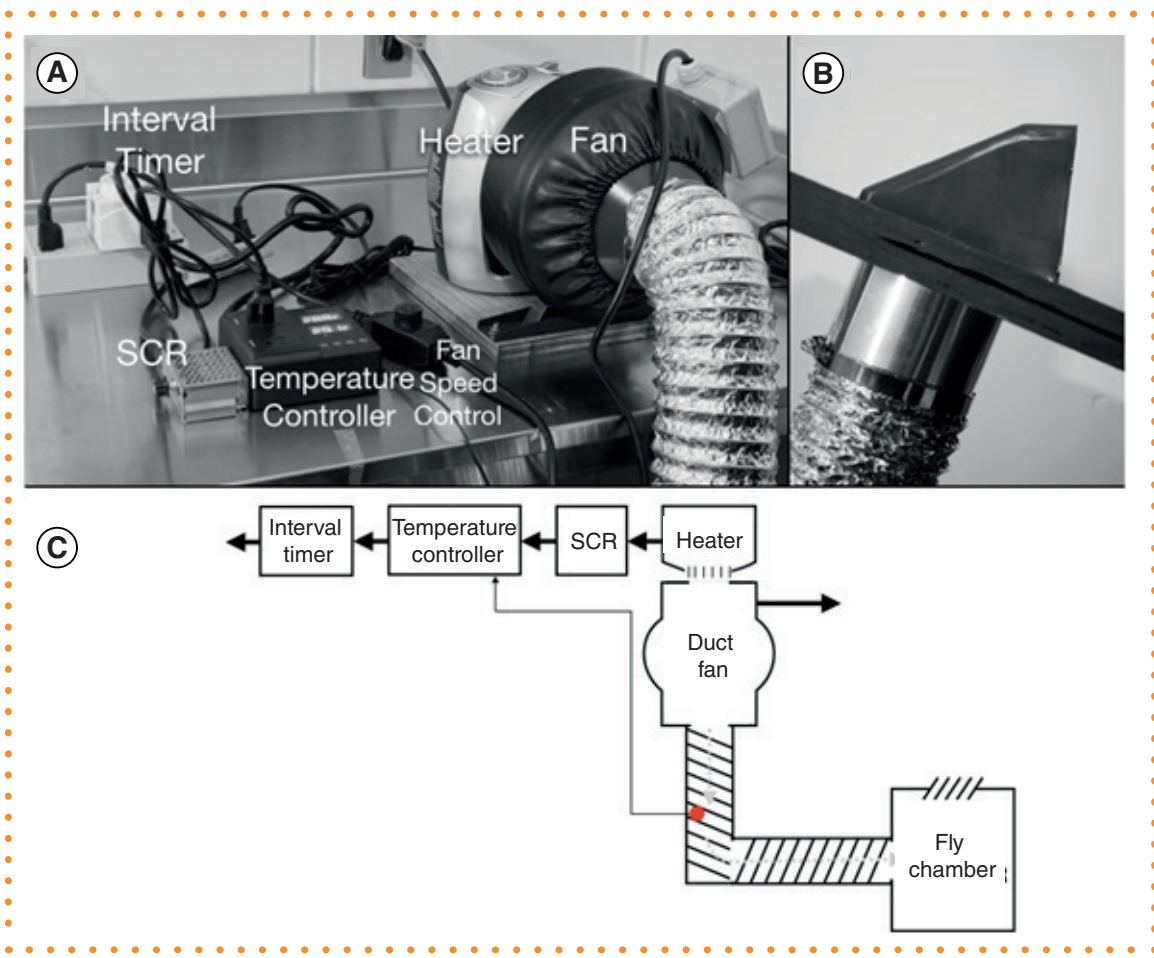

Figure 1. Image $(A)$ and schematic of the air stream temperature controller $(C)$, and connection to the chamber holding the flies (B). (A) The heat source is an office floor heater, controlled by an interval timer that powers a temperature controller. The power output from the temperature controller is limited by a SCR, to reduce temperature overshoot. The probe (red dot) for the temperature controller is placed within the ducting. The fan is powered continuously. Arrows indicate male power plugs. The equipment listed is readily available from online retail vendors. (B) The front door of the fly chamber, edge on and tilted open, with a dryer vent allowing entrance of the temperature-conditioned airstream. SCR: Silicone-controlled rectifier.

- generally being a positive regulator of locomotor activity $[14,15]$, these flies show significant increase in activity versus controls from approximately the second to the tenth minute at $29^{\circ} \mathrm{C}$, at which point it decays gradually well before the end of the 20 min interval at $29^{\circ} \mathrm{C}$. This decrement in locomotor activity extends into the subsequent downshift to $22^{\circ} \mathrm{C}$, where these flies exhibit lower activity than the controls. A reasonable explanation for these effects is that $\mathrm{dTrpA} 1$ activation is depleting intracellular stores of dopamine, an explanation that is currently under investigation.

Our system is adaptable to other temperature-dependent assays. For example, the digital temperature controller used here has an option for powering a cooling circuit, opening possibilities for more rapid and controlled temperature downshifts. Also, the silicone-controlled rectifier (SCR) can be adjusted to change the voltage to the heater. This will change the kinetics of the system such that heating can be modulated faster or slower based on biological requirements.

\section{FLY HOUSING \& TEMPERATURE CONTROL}

Parts used are listed in Table 1. Flies, in 5-mm OD tubes mounted in DAM2 Activity Monitors (Trikinetics, MA, USA), were housed in a custom built $0.13 \mathrm{~m}^{3}$ light-tight wooden fly chamber of inner dimensions $54 \mathrm{~W} \times 54 \mathrm{D} \times 45 \mathrm{~cm} \mathrm{H}$, in a temperature/ humidity-controlled walk-in environmental room (EGC, OH, USA) set at $21^{\circ} \mathrm{C}$ and $60 \%$ relative humidity $[16,17]$. The $\mathrm{EGC}$ room was modified to replace the squirrel cage fan coil motors with rotary fans, since the squirrel cage fans generated a strong $120 \mathrm{~Hz}$ vibration that affected behavior of the flies.

The light-tight wooden chamber has a light-tight baffled side vent and a front door that opens via a piano hinge. It can hold $\sim 20$ fly activity monitors, each capable of monitoring 32 flies. Temperature within the chamber was monitored in real time using a Temperature Alert monitoring system (no longer sold), or with a Hobo temperature monitor (Onset Computer Corporation, MA, USA) set to monitor temperature at $1 \mathrm{~min}$ intervals. Data from the Hobo temperature monitor is not shown and it is slower to respond than the Temperature Alert system.

\section{Construction of the air stream temperature controller}

The only critical mechanical coupling is of the space heater to the fan (Figure 1A). These components are mounted on a $10 \times 10$ " piece of $3 / 4^{\prime \prime}$ plywood. The fan is mounted with $1 / 4^{\prime \prime} \times 20$ bolts and the heater is mounted adjacent to the fan inlet using double-sided outdoor mounting tape (Scotch, \#4011, MN, USA), but not otherwise physically coupled to the fan. With this arrangement, the fan efficiently captures the warmed air. Temperature-conditioned air was ported through the front door of the wooden chamber holding the flies via a clothes dryer vent (Figure 1B) that penetrates the hinged front door through a 4 " hole.

\section{Logic of the circuit}

A schematic of the controller is shown in Figure 1C. An interval timer controls off/on intervals of the heater circuit by feeding power to the proportional temperature controller. The temperature controller output goes to an SCR, which reduces the voltage to the heater, thus limiting temperature overshoot. In our setup, the space heater is set on its low setting, 750 watts, but with the thermostat set at just past halfway.

The Bayite temperature controller is set at $31.3^{\circ} \mathrm{C}$ with the heating differential set at $0.1^{\circ} \mathrm{C}$. The exact set point and heating differential will vary between individual setups as a function of duct length, ambient temperature and other variables that affect heat loss in the duct. The SCR is set at $~ 82$ VAC, such that the wattage of the heater is $\sim 510$ watts. The temperature probe of the controller is mounted at the output of the fan within the duct.

The only electrical connections that need to be modified are those to and from the SCR, which has screw terminals for input and output. This was done by cutting a power strip cord, connecting the wire with the male plug to the SCR inputs, and the female socket end to the SCR outputs, while maintaining a common ground between all components. Wiring of power connections should be done by qualified/licensed personnel.

Heat generated by the fan motor is sufficient to raise the temperature in the 
fly chamber $\sim 1^{\circ} \mathrm{C}$ from ambient temperature. The probe for the temperature controller should be placed in the ducting near the output from the fan to permit rapid responses. Due to heat loss in the duct, a $\sim 2^{\circ} \mathrm{C}$ temperature difference was measured from the probe within the chamber and the probe from the temperature controller. Therefore, the temperature controller should be adjusted to maintain the $29^{\circ} \mathrm{C}$ setpoint within the chamber rather than relying on the set value and heat differential.

Activity data from the Trikinetics monitors was analyzed using a custom $R$ script that averages 5 days of data at each time point and plotted using ggplot2. A one-way ANOVA was calculated at each time point with an alpha of 0.05 . At points where a significant difference was detected, a Tukey's honest significance difference post hoc test was performed (Figure 2). Asterisks indicate time points at which driven flies are significantly different from both controls. Temperature data was also analyzed in R. 8 days of temperature data were averaged at each minute for the indicated temperature shifts.

\section{AUTHOR CONTRIBUTIONS}

Ryan Sangston and Jay Hirsh both contributed significantly to experiment design, interpretation of data, and drafting of the document. Ryan Sangston was responsible for the data acquisition and analysis.

\section{FINANCIAL \& COMPETING INTERESTS DISCLOSURE}

Supporting grant: NIH 5R01 GM084128. The authors have no other relevant affiliations or financial involvement with any organization or entity with a financial interest in or financial conflict with the subject matter or materials discussed in the manuscript apart from those disclosed.

No writing assistance was utilized in the production of this manuscript.

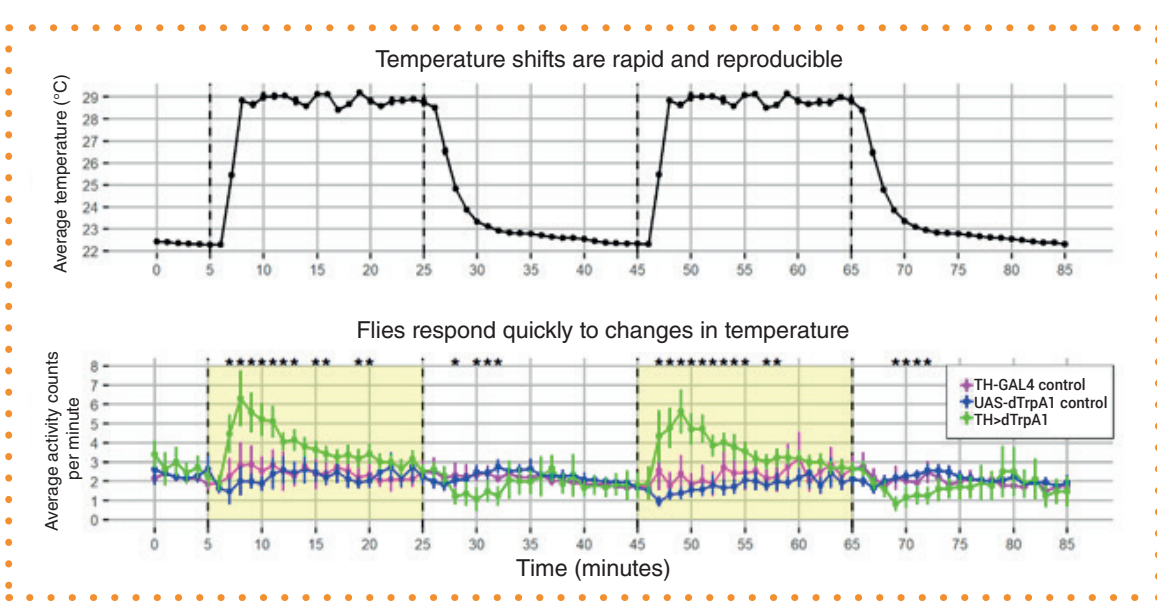

Figure 2. Rapidly induced temperature changes elicit rapid responses from flies expressing dTrpA1 in TH neurons. (Top) Each time point is an average of eight data points at that point in time across eight different days. Dotted vertical lines indicate when the heater was turned on or off by the programmable timer. Error bars are SEM. (Bottom) Intervals when the heater is activated are highlighted in yellow. By the second minute, TH driven dTrpA1 flies have increased activity levels and the timing coincides with the measured temperature increase. TH-GAL4 control $n=31$; UAS-dTrpA1 control $n=32$; TH > dTrpA1 $n=32$.

Asterisks indicate a significant difference between driven and control flies where $p<0.05$ as calculated by a one-way ANOVA followed by Tukey's honest significance difference post hoc test.

\section{OPEN ACCESS}

This work is licensed under the AttributionNonCommercial-NoDerivatives 4.0 Unported License. To view a copy of this license, visithttp:// creativecommons.org/licenses/by-nc-nd/4.0/

\section{REFERENCES}

1. Yoshihara M, Ito K. Acute genetic manipulation of neuronal activity for the functional dissection of neural circuits - a dream come true for the pioneers of behavioral genetics. Neurogenet. 26(1), 43-52 (2012).

2. Kitamoto T. Conditional modification of behavior in Drosophila by targeted expression of a temperature-sensitive shibire

3. Matsumoto K, Toh-e A, Oshima Y. Genetic control of galactokinase synthesis in Saccharomyces cerevisiae: evidence for constitutive expression of the positive regulatory gen gal4. J. Bacteriol. 134(2), 446-457 (1978).

4. Brand AH, Perrimon N. Targeted gene expression as a means of altering cell fates and generating dominant phenotypes. Development 118(2), 401-415 (1993).

5. Hamada FN, Rosenzweig M, Kang K et al. An internal thermal sensor controlling temperature preference in Drosophila. Nature 454(7201), 217-220 (2008).

6. Brand M, Jarman AP, Jan LY, Jan YN. asense is a Drosophila neural precursor gene and is capable of initiating sense organ formation. Development 119(1), 1-17 (1993).

7. Aso Y, Siwanowicz I, Bracker L, Ito K, Kitamoto T, Tanimoto $\mathrm{H}$. Specific dopaminergic neurons for the formation of labile aversive memory. Curr. Biol. 20(16), 1445-1451 (2010).

8. Berry JA, Cervantes-Sandoval I, Nicholas EP, Davis RL. DoNeuron. 74(3), 530-542 (2012)
9. Chung BY, Ro J, Hutter SA et al. Drosophila neuropeptide F signaling independently regulates feeding and sleep-wake behavior. Cell Rep. 19(12), 2441-2450 (2017).

10. Liu Q, Liu S, Kodama L, Driscoll MR, Wu MN. Two dopaminergic neurons signal to the dorsal fan-shaped body
to promote wakefulness in Drosophila. Curr. Biol. 22(22) 2114-2123 (2012).

11. Robie AA, Hirokawa J, Edwards AW et al. Mapping the neural substrates of behavior. Cell 170(2), 393-406.e328 (2017).

12. Lamaze A, Ozturk-Colak A, Fischer R, Peschel N, Koh K, Jepson JE. Regulation of sleep plasticity by a thermo-sensitive son JE. Regulation of sleep plasticity by a the
circuita. Sci. Rep. 7, 40304 (2017).

13. Friggi-Grelin F, Coulom H, Meller M, Gomez D, Hirsh J, Birman S. Targeted gene expression in Drosophila dopaminergic cells using regulatory sequences from tyrosine hydroxylase. J. Neurobiol. 54(4), 618-627 (2003).

14. Andretic R, van Swinderen B, Greenspan RJ. Dopaminergic modulation of arousal in Drosophila. Curr. Biol. 15(13) 1165-1175 (2005).

15. Kume K, Kume S, Park SK, Hirsh J, Jackson FR. Dopamine is a regulator of arousal in the fruit fly. J. Neurosci. 25(32), 7377-7384 (2005).

16. Hirsh J, Riemensperger T, Coulom H, Iche M, Coupar J, Birman S. Roles of dopamine in circadian rhythmicity and extreme light sensitivity of circadian entrainment. Curr. Biol. 20(3), 209-214 (2010)

17. Vinayak $P$, Coupar J, Hughes SE et al. Exquisite light sensi9(7), e1003615 (2013).

\section{Table 1. Fly housing \& temperature control equipment used.}

\begin{tabular}{|l|l|}
\hline Fan (and fan speed control) & VIVOSUN 4 inch 203 CFM inline duct ventilation fan with variable speed controller \\
\hline Fan ducting & 4 inch diameter $\mathrm{x} 8 \mathrm{ft}$ non-insulated flex air aluminum ducting dryer vent hose \\
\hline Heater & AmazonBasics 1500 Watt Ceramic Space heater with adjustable thermostat \\
\hline Interval timer & Century 7 day heavy duty digital programmable timer - dual outlet (single control) \\
\hline Adjustable voltage regulator (SCR) & Motor speed controller, DROK motor control board AC 110V 4000W adjustable voltage regulator SCR \\
\hline Temperature controller & Bayite temperature controller 1650W BTC211 digital outlet thermostat \\
\hline
\end{tabular}

